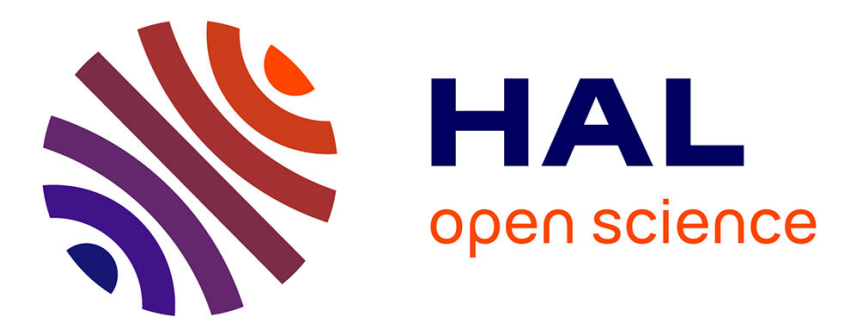

\title{
A hydrodynamic analogy for shearing stress distribution in bending
}

\author{
Maurice A. Biot
}

\section{To cite this version:}

Maurice A. Biot. A hydrodynamic analogy for shearing stress distribution in bending. Journal of Applied Physics, 1938, 9 (1), pp.39-43. 10.1063/1.1710358 . hal-01368609

\section{HAL Id: hal-01368609 \\ https://hal.science/hal-01368609}

Submitted on 13 Oct 2016

HAL is a multi-disciplinary open access archive for the deposit and dissemination of scientific research documents, whether they are published or not. The documents may come from teaching and research institutions in France or abroad, or from public or private research centers.
L'archive ouverte pluridisciplinaire HAL, est destinée au dépôt et à la diffusion de documents scientifiques de niveau recherche, publiés ou non, émanant des établissements d'enseignement et de recherche français ou étrangers, des laboratoires publics ou privés. 


\author{
REPRINT SERIES
}

A HYDRODYNAMIC ANALOGY FOR SHEARING STRESS DISTRIBUTION IN BENDING

BY

M. A. BIOT

Reprinied from Journat or Applien Puysics, Vol.9, No. 1, January, 1938 
Reprinted from Journal of Applied Physics, Vol. 9, No. 1, January, 1938

Copyright 1937 by the American Institute of Physics

Printed in U. S. A.

\title{
A Hydrodynamic Analogy for Shearing Stress Distribution in Bending*
}

\author{
M. A. BIOT \\ Columbia University, New York, N.Y.
}

\begin{abstract}
It is shown here that the shearing stress distribution in the combined shear and bending is represented with practical accuracy by the distribution of velocity in the flow of a perfect fluid over the area of the cross section. This flow is produced by a linear distribution of sources above the neutral axis and of sinks below the neutral axis, the intensity of these sources and sinks being proportional to the distance to the neutral axis. For hollow beams the additional condition must be added that the circulation of the velocities is zero around each hole of the cross section. The analogy which is rigorous for a material of zero Poisson ratio holds within a small correction for the general case.
\end{abstract}

$\mathbf{I}^{\mathrm{T}}$ was established by Saint-Venant from the mathematical theory of elasticity that the combination of bending and shear in a uniform beam of arbitrary cross section yields a very simple distribution of the bending stresses, which coincides with the one derived from elementary strength of materials. The shearing stresses, however, do not obey such a simple law and the usual mathematical treatment does not yield easily a fairly approximate idea of their distribution.

Our purpose is to show that the shearing stress distribution can be visualized by means of a rather simple physical analogy and furthermore that the fundamental equations at the base of this analogy may be reached directly by a shortcut method.

\section{The Generat Case of Combinfen Bending SHEAR AND ToRsion}

We consider a uniform beam ( $F$ ig. 1) referred to a system of rectangular coordinates $x, y, z$. The $z$ axis is parallel with the beam, the $x$ axis passes through the center of gravity $G$ of the cross section and coincides with one of the principal axes of inertia of this cross section. The location of the origin along the $x$ axis is

* Publication assisted by the Ernest Kempton Adams Fund for Physical Research of Columbia University.

left arbitrary. As will be shown hereafter a system of stress components $\sigma_{z} \tau_{x} \tau_{y}$ (Fig. 1) is compatible with the theory of elasticity and may be selected in such a manner as to result in the combination of a bending moment $M$ and a total shear $S$ perpendicular to $x$.

We impose upon the stresses the further restrictions :

(1) That $\sigma_{z}$ has a linear distribution along $z$ and $y$ by putting

$$
\sigma_{z}=(S / I) y z .
$$

$I$ is the moment of inertia of the cross section with respect to the $x$ axis.

(2) That the shearing stress $\left(\tau_{x}, \tau_{y}\right)$ acting on the cross section be independent of the location of this cross section, i.e. independent of $z$.

The above stress system, in order to be compatible with the theory of elasticity, must verify the following set of equations:

(1) The generalized Hooke's law

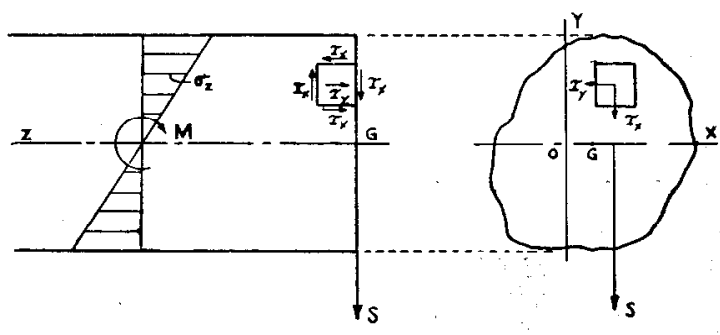

FIG. 1. 


$$
\begin{aligned}
\partial u / \partial x & =-(\mu / E I) S y z, \\
\partial v / \partial y & =-(\mu / E I) S y z, \\
\partial w / \partial z & =(S / E I) y z, \\
\partial u / \partial y+\partial v / \partial x & =0, \\
\partial u / \partial z+\partial w / \partial x & =\tau_{y} / G \\
\partial v / \partial z+\partial w / \partial y & =\tau_{x} / G .
\end{aligned}
$$

(2) The condition of internal equilibrium

$$
\partial \tau_{x} / \partial y+\partial \tau_{y} / \partial x=-\partial \sigma_{z} / \partial z=-(S / I) y .
$$

In these equations $u, v, w$ are the coordinate increments; the original coordinates $x, y, z$ of a

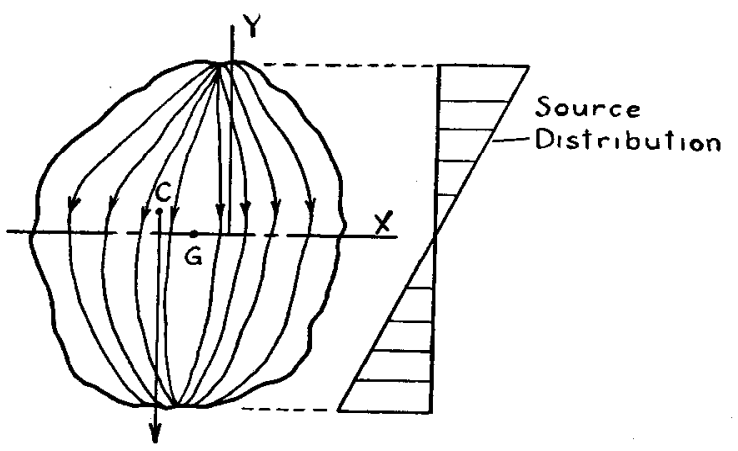

FIG. 2.

point attached to the material become $(x+u)$, $(y+u),(z+w)$ after deformation.

$E$ is the elasticity modulus, $G$ the shear modulus and $\mu$ the Poisson ratio of the material.

It can be readily verified that Eqs. (2), (3), (5) become identities and that the other Eqs. (4), (6), (7) are compatible if we put

$$
\begin{gathered}
u=\partial f / \partial x, \quad v=-\partial f / \partial y, \\
f=\left((S / 6 E I) y z^{3}\right)+\left((\mu S / 6 E I) z y^{3}\right) \\
-\left((\mu S / 2 E I) x^{2} y z\right)+\omega x y z,
\end{gathered}
$$

where $\omega$ is a constant to be determined later. ${ }^{1}$

Since we are primarily interested in the distribution of $\tau_{x}$ and $\tau_{y}$ we now proceed to eliminate $w$ between (6) and (7). Taking into account (9) and (10) we find

$\partial \tau_{y} / \partial y-\partial \tau_{x} / \partial x=-(\mu /(1+\mu))(S / I) x+2 G \omega$.

This last equation with the condition of

\footnotetext{
1 Form (10) of $f$ is not the most general that can be found. However, it only differs from this general form by terms which correspond to a displacement of the whole beam as a solid body.
}

equilibrium (8) solves our problem. It is convenient to introduce the two stress functions $\varphi$ and $\psi$ in the following way.

$$
\begin{aligned}
& \tau_{y}=-\partial \varphi / \partial x-\partial \psi / \partial y, \\
& \tau_{x}=-\partial \varphi / \partial y+\partial \psi / \partial x .
\end{aligned}
$$

From (8) and (11) we deduce that these two stress functions verify the following equations,

$$
\left(\partial^{2} / \partial x^{2}+\partial^{2} / \partial y^{2}\right) \varphi=(S / I) y,
$$

$\left(\partial^{2} / \partial x^{2}+\partial / \partial y^{2}\right) \psi=(\mu /(1+\mu))(S / I) x-2 G \omega$.

We also have as boundary condition that the shearing stress is tangent to the contour of the cross section

$$
\begin{gathered}
\tau_{y} d y-\tau_{x} d x=0 \\
\text { or } \quad(\partial \varphi / \partial x) d y-(\partial \varphi / \partial y) d x-d \psi=0 .
\end{gathered}
$$

We may split this condition into the following two:

$$
\begin{aligned}
(\partial \varphi / \partial x) d y-(\partial \varphi / \partial y) d x & =0, \\
\psi & =\text { const. }
\end{aligned}
$$

From Eqs. (13) and (14) it appears that the shearing stress distribution is made of the superposition of the following systems of stress (a) and (b) one being represented by a hydrodynamic analogy and the other by a membrane analogy.

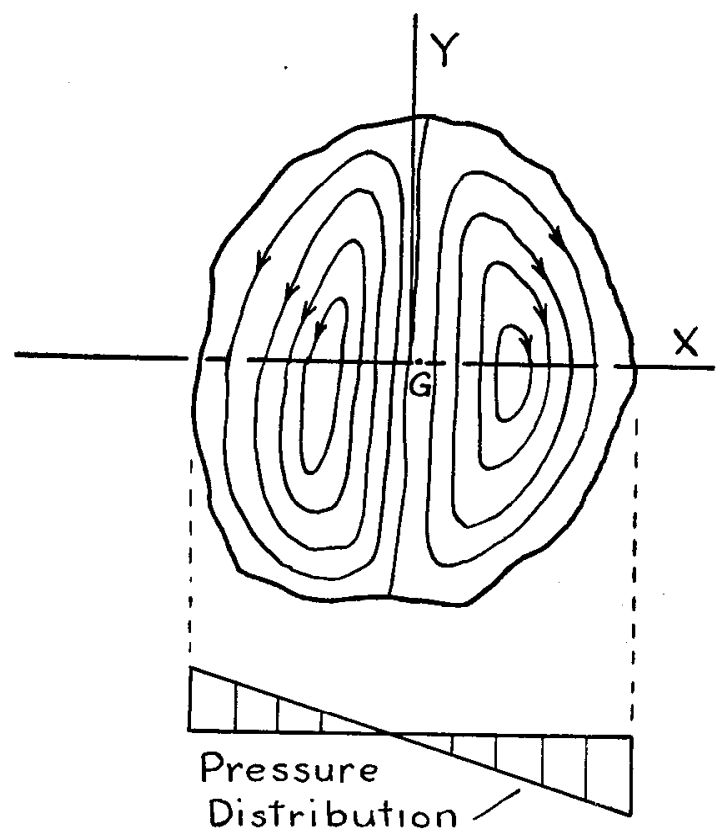

FIG. 3.

Journal of APPLIEd Physics 


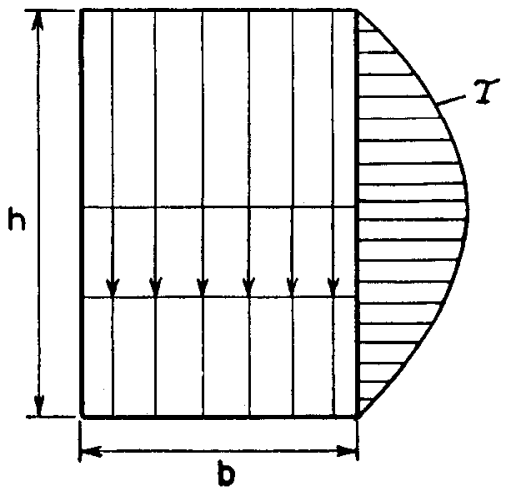

FIG. 4.

(a) One is the gradient of the function $\varphi(x, y)$. Eqs. (13) and (15) show that it may be represented by the velocity distribution in the two-dimensional flow of a perfect fluid with sources of intensity $(S / I) y$ per unit area. The function $\varphi(x, y)$ is the hydrodynamic potential of this flow. Above the $x$ axis we have positive sources of intensity proportional to their distance to this axis, and below the $x$ axis we have negative sources of intensity also proportional to their distance to this axis. Since we have assumcd the $x$ axis to pass through the center of gravity of the section, the total flow out of the sources equals the total flow disappearing in the sinks.

(b) The other part of the stress defined by the stress function $\psi(x, y)$ depends on the Poisson ratio. It may be represented by the membrane analogy. The membrane is stretched so as to rest on a horizontal plane boundary the contour of which is the same as that of the beam section. The membrane is submitted on one side to a pressure $p$ distributed linearly in a direction parallel with the neutral axis according to the law $p=-(\mu /(1+\mu))(S / I) x+2 G \omega$. The stress is equal to the slope of this membrane and tangent to the contour lines of equal height. The local rotation of the section is $-(1 / 2 G)(\mu /(1+\mu))$ $\times(S / I) x+\omega$. Both this rotation and the stresses (b) represented by the membrane depend on the choice of the constant $\omega$ and the location of the origin on the $x$ axis.

It can be proved as in the theory of the membrane analogy for pure torsion that the (b) stresses result in a torque of value equal to twice the volume under the membrane.
11. 'The Case of Pure Shear and Bending

Let us first assume that the Poisson ratio $\mu=0$. In that case by choosing $\omega=0$ the stress system (b) disappears and there is no rotation of the cross section. This is a case of pure shear and bending. The shear stresses are rigorously determined by the hydrodynamic analogy, and they result into a total shear $S$ applied along a definite line of action perpendicular to the $x$ axis. We could also find in the same way the line of action of a pure shear acting in a direction parallel with the $x$ axis. The point of intersection of these two lines of action defines the center of shear $C$ (Fig. 2).

If the Poisson ratio is not zero a force acting through the center of shear produces not only the above stress system (a), but also a certain stress system (b). For instance if the total shear acts through the center of shear $C$, the corresponding system (b) will be found by choosing

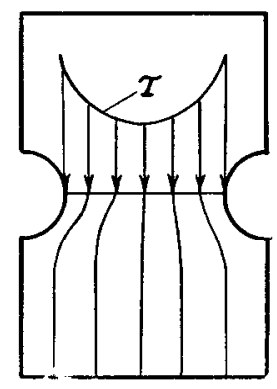

FIG. 5.

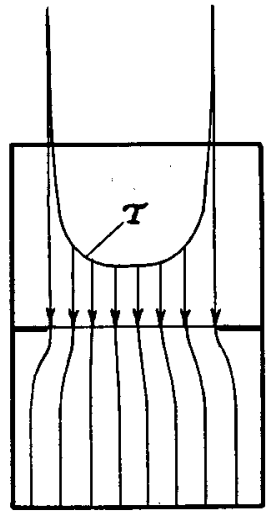

FIG. 6 the constant $\omega$ so that the system will be composed of two equal and opposite lorques. In terms of the membrane analogy this means that we must choose the zero pressure line so as to make the amount of positive volume under the membrane equal to the amount of negative volume. This additional system (b) appears as a correction to (a) when the effect of lateral contraction is taken into account. It originates from the fact that the lateral contraction due to the existence of the normal bending stresses on the cross section varies from one section to the other, thus changing the shape of these sections 
relative to one another and introducing additional shears and rotations (Fig. 3). ${ }^{2}$

This effect will in general be very small and it is justified to neglect the influence of the lateral contraction in the applications and to take only into account the stresses given by the hydrodynamic analogy. We note that when $\mu$ is not zero there is no such thing as really pure shear since there will always be a rotation whatever the location of the resultant shear $S$.

\section{Examples for Solid Beams}

(1) Rectangular cross section (Fig. 4). The flow lines are vertical and the velocity is calculated from the value of the total flow. The stress $\tau$ being at a distance $y$ from the neutral axis we have

$$
\begin{aligned}
b \tau & =\int_{y}^{h / 2}(S y / I) b d y, \\
\tau & =(S / 2 I)\left[(h / 2)^{2}-y^{2}\right] .
\end{aligned}
$$

It is the well-known parabolic distribution. From the theory above it appears that this is rigorously correct when $\mu=0$ and when $\mu \neq 0$ the correction is generally very small, so that it can be considered as a very good approximation in the latter case.

(2) Section with a fillet. It is known that the effect of a semi-circular fillet on potential flow is to double the velocity of the fluid at the fillet. Such a fillet will be expected to introduce a stress concentration factor of about two (Fig. 5). For a section with a sharp slit the total shear

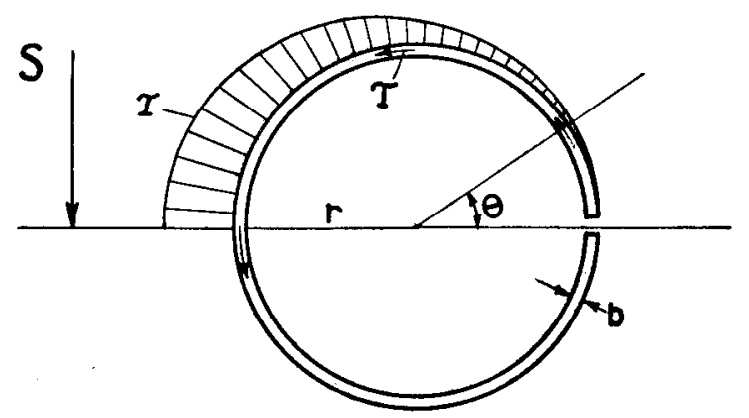

FIG. 7.

2 The separation of the shearing stresses in these two systems has also been considered by $\mathrm{C}$. Weber, "Biegung und Schub in geraden Balken," Zeits. f. angew. Math. u. Mech. 334-348 (1924). will be infinite at the slit. In practice this will produce local plastic flow (Fig. 6).

(3) Open thin section. For a circular thin tube (Fig. 7) of thickness $b$ cut open along its length the flow at an angle $\theta$ is

$$
\begin{gathered}
\delta \tau=\int_{0}^{\theta}(S r \sin \theta / I) b r d \theta, \\
\tau=\left(S r^{2} / I\right)(1-\cos \theta) . \\
\text { IV. HoLLOW BEAMS }
\end{gathered}
$$

In a hollow beam the cross section contains one or more holes. The hydrodynamic potential $\varphi$ is not necessarily single-valued since an arbitrary circulation may be added around each hole. Moreover the value of the function $\psi$ is not given along the boundary. It has only been derived in the general theory that $\psi$ must be a constant along one boundary contour, but this

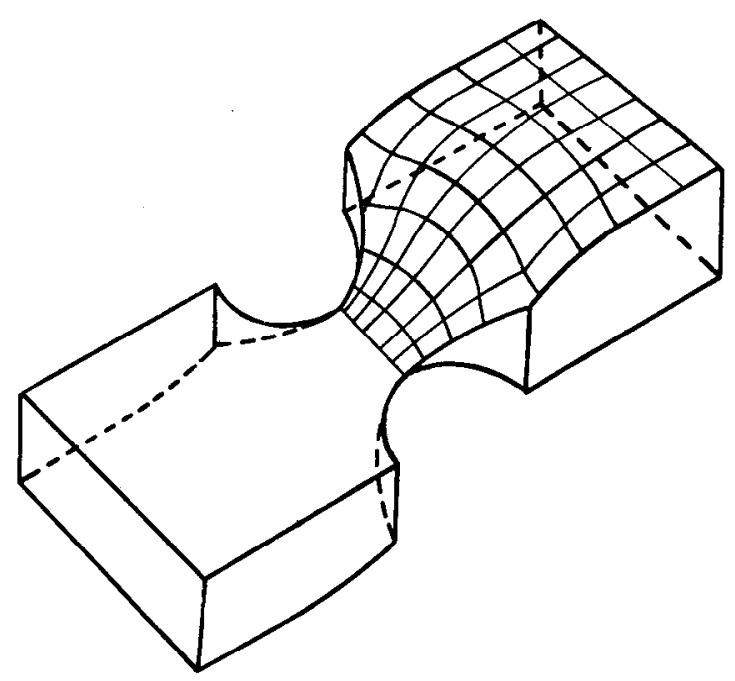

FIG. 8.

constant may be different for each different contour.

In order to determine the problem we must introduce the extra condition that the displacement $w$ will be single-valued. We have

$$
d w=(\partial w / \partial x) d x+(\partial w / \partial y) d y .
$$

Using relations $(6),(7),(9),(10)$, this becomes

$$
\begin{aligned}
d w=(1 / G)\left(\tau_{y} d x+\tau_{x} d y\right) & -\left(\left(\partial^{2} f / \partial x \partial z\right) d x\right)+\left(\left(\partial^{2} f / \partial y \partial z\right) d y\right) .
\end{aligned}
$$

The function $w(x, y)$ will be single-valued if 


$$
\oint_{C} \tau_{y} d x+\tau_{x} d y=\oint_{C} G\left(\frac{\partial^{2} f}{\partial x \partial z} d x-\frac{\partial^{2} f}{\partial y \partial z} d y\right),
$$

where these integrals are taken on a closed contour $C$ around each hole. The right-hand side of this equation may be expressed as a double integral extended to the area $D$ inside the contour $C$ and we may write condition (20) as

$$
\begin{aligned}
\oint_{C} \tau_{y} d x+\tau_{x} d y \\
\left.\quad=\iint_{D}(1 \mu /(1+\mu))(S / I) x-2 G \omega\right) d x d y
\end{aligned}
$$

Considering now relation (12) this last condition may be replaced by the following two

$$
\begin{aligned}
& \oint_{\mathrm{C}}(\partial \varphi / \partial x) d x+(\partial \varphi / \partial y) d y=0, \\
& \oint_{C}(\partial \psi / \partial y) d x-(\partial \psi / \partial x) d y \\
& =\iint_{D}((-\mu /(1+\mu))(S / I) x+2 G \omega) d x d y .
\end{aligned}
$$

This amounts to stating that the stress system (a) given by the hydrodynamic analogy and the stress system (b) due to the lateral contraction considered separately both yield a single-valued displacement $w$.

Condition (21) expresses that the hydrodynamic potential must be single-valued, i.e., the circulation around each hole must be zero. Here again it is legitimate to assume that in most cases the stress due to the lateral contraction is negligible and to consider as a good approximation the system of stresses represented by the hydrodynamic analogy with the condition that the circulation is zero.

\section{Remarks Regarding the Elastica and the Distortion of the SEction}

From (9) and (10) we deduce for the vertical displacement of the points originally on the $z$ axis

$$
v=-(S / 6 E I) z^{3} .
$$

This coincides with the shape of the elastica found by elementary strength of materials.

It must be added that in practice this is not the only deformation that occurs since we must also take into account the local effect of the distribution of stress at the boundaries and around the points of application of the load. A small additional deflection depending only on this local effect will have to be added, and it is to be expected that this additional value is roughly proportional to the total shear.

It is quite obvious that a cross section does not remain plane after the deformation of the beam. The amount of distortion and the shape of the distorted section may easily be deduced from the hydrodynamic analogy if we neglect the effect of the lateral contraction.

Assuming $\mu=0, \omega=0$ in (10) and the consequence $\psi=0$ we obtain from (6) and (7)

$$
\begin{aligned}
\partial w / \partial x & =-(1 / G)(\partial \varphi / \partial x), \\
-(S / 2 E I) z^{2}+\partial w / \partial y & =-(1 / G)(\partial \varphi / \partial y) .
\end{aligned}
$$

We conclude

$$
w=-(1 / G) \varphi+(S / 2 E I) z^{2} y .
$$

The second term represents the rotation of the section as a plane perpendicular to the elastica, hence the first represents the distortion. Hence the distortion is represented by the hydrodynamic potential.

By use of this result the distortion of a rectangular section with circular fillet is represented in Fig. 8. 